\title{
Meta-analysis of whole-transcriptome data suggests new mechanisms of auxin-induced ethylene biosynthesis and signaling in Arabidopsis thaliana
}

\author{
E. Ubogoeva \\ Novosibirsk State University, Novosibirsk, Russia \\ e-mail:ubogoeva@gmail.com
}

Key words: auxin-ethylene crosstalk, RNA-seq, microarray

Motivation and Aim: Plant hormones auxin and ethylene are the key regulators of plant growth and development, which are widely used in agriculture. The crosstalk between these hormones is essential for regulation of many physiological processes. Ethylene often achieves its morphogenetic effects by modulating auxin biosynthesis and transport and thereby changing the distribution of auxin - the major regulator of plant morphogenesis [1]. This crosstalk is reciprocal as auxin induces ethylene biosynthesis. However, the mechanisms of auxin effects on ethylene metabolic and signaling pathways is still not clear. Here we perform meta-analysis of auxin-induced whole-transcriptome data to unveil the molecular mechanisms of auxin-induced ethylene biosynthesis and signaling in Arabidopsis thaliana root.

Methods and Algorithms: We used our own RNA-seq data and publicly available microarray data [2] on auxin-induced transcriptomes of A. thaliana root for metaanalysis. Differentially expressed genes were filtered based on Gene Ontology (GO) term inherence. We used the binomial trail estimate to separate the robust predictions. We used hierarchical clustering to find co-expression patterns.

Results: We identified 128 auxin-responsive genes, which were associated with ethylene biosynthesis, signaling or ethylene response. Auxin sensitivity of these genes was selectively verified by qRT-PCR. The auxin sensitivity of some genes was shown for the first time. We also predicted several primary ARF targets. We further determined specific auxin-induced expression profiles for ethylene biosynthesis genes. Based on the data obtained, a scheme of auxin-dependent regulation of ethylene biosynthesis and transcriptional response was proposed.

Conclusion: Due to increasing number of publicly available high-throughput genomic data meta-analysis is a powerful approach for the genome-wide screening.

Acknowledgements: Supported by Russian Foundation for Basic Research and the government of Novosibirsk region, grant No. 18-44-540039.

\section{References}

1. Muday G.K., Rahman A., Binder B.M. (2012) Auxin and ethylene: Collaborators or competitors? Trends Plant Sci. 17(4):181-195.

2. Lewis D.R., Olex A.L., Lundy S.R., Turkett W.H., Fetrow J.S., Muday G.K. (2013) A kinetic analysis of the auxin transcriptome reveals cell wall remodeling proteins that modulate lateral root development in Arabidopsis. Plant Cell. 25(9):3329-3346. 